\title{
Integrating Ecoinformatics Resources on the Semantic Web
}

Cynthia Sims Parr
csparr@umbc.edu

\author{
Andriy Parafiynyk \\ andr1@umbc.edu
}

Joel Sachs

jsachs@umbc.edu
Li Ding

ding.li@umbc.edu
Sandor Dornbush

sandor1@umbc.edu
Tim Finin

finin@umbc.edu

Department of Computer Science and Electrical Engineering

University of Maryland Baltimore County

Baltimore, MD 21250 USA

\author{
David Wang \\ Dept. of Computer Science \\ University of Maryland \\ College Park, MD, 20742 USA \\ Tw7@cs.umd.edu
}

\author{
Allan Hollander \\ Information Center for the Environment \\ University of California Davis \\ Davis, CA, 95616 USA \\ adhollander@ucdavis.edu
}

\begin{abstract}
We describe ELVIS (the Ecosystem Location Visualization and Information System), a suite of tools for constructing food webs for a given location. We express both ELVIS input and output data in OWL, thereby enabling its integration with other semantic web resources. In particular, we describe using a Triple Shop application to answer SPARQL queries from a collection of semantic web documents. This is an end-to-end case study of the semantic web's utility for ecological and environmental research.
\end{abstract}

\section{Categories and Subject Descriptors}

H. Information Systems

H.4 Information System Applications

H.4.m miscellaneous

\section{General Terms}

Algorithms, Design, Experimentation, Human Factors, Standardization,

\section{Keywords}

Food webs - Ecological forecasting - Semantic web - Ontologies - Invasive Species - Biodiversity - Service Oriented Design

\section{INTRODUCTION}

SPIRE (Semantic Prototypes in Research Ecoinformatics $\mathrm{http}: / /$ spire.umbc.edu) is a distributed, interdisciplinary research project tasked with building semantic web prototypes for invasive species science.

Our main integrating suite of tools is ELVIS (the Ecosystem Location Visualization Information System). ELVIS is motivated by the belief that food web structure plays a role in the success or failure of potential species invasions. Because very few ecosystems have been the subject of empirical food web studies, response teams are typically unable to get quick answers to questions like "what are likely prey and predator species of the invader in the new environment?"

The core data has been integrated from publicly available sources and is now available on the semantic web. We have constructed a platform for investigating multiple algorithms for food web prediction. Further, by exposing item-level data through several rich sets of ecological and evolutionary ontologies, and by providing these tools as web services, we enable integration with other semantic web/web 2.0 applications, such as Swoogle. We developed a "shopping cart" application, Triple Shop, which allows a user to select semantic web documents, and to issue SPARQL queries over their union. Thus, we are able to integrate diverse ecoinformatics data in response to ad-hoc queries.

\subsection{Related Work}

Previous work on data integration in ecological informatics includes online data repositories [2] and workflow [4] ontologies. Metadata allows only the discovery of possibly interesting datasets and does not provide the means to harvest the data itself. Individual food web researchers maintain and share their own digital data archives, in individualized data formats, though more accessible standardized archives are beginning to emerge [1]. There are good databases on invasive species (e.g. http://www.issg.org/) but they are not automatically integrated with information about non-invasive species with which they interacy; nor is there web-based support for modeling an invasive species, anywhere.

\section{ELVIS}

The task of providing food web information for a user-specified location breaks into two distinct problems: constructing a species list for a given location; and constructing a food web from a given species list (and habitat information).

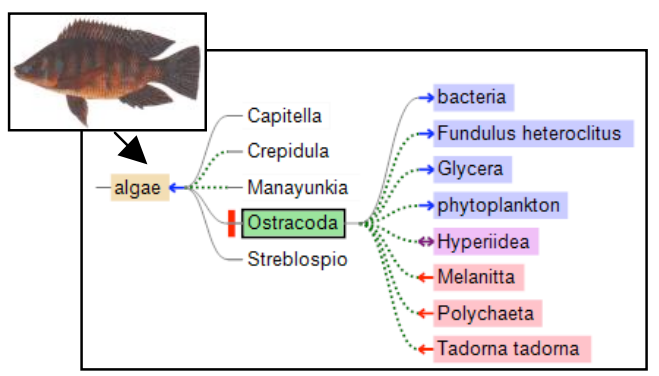

Figure 1. Nile Tilapia, an invader in Florida ecosystems, is predicted to eat algae and have no potential predators. Organisms predicted to be nearby in the food web (to the right of algae) could be impacted by or mediate the introduction of this competitor.

\subsection{Species List Constructor}

Our goal is to allow a user to input a location, and get back a species list for that location. This is a hard problem, typically adhoc, and relying on expert knowledge. There are, in general, three kinds of information that can be used to generate a species list: (i) park inventories; (ii) point locations, e.g. from specimen descriptions in museums and herbariums; and (iii) distribution 
maps generated by applying statistical techniques to point locations. We are integrating all of the above for California, and expect that the ontologies and synthesis strategies we have developed will apply to other states, and enable ELVIS to quickly spread beyond California.

\subsection{Food Web Constructor}

The Food Web Constructor (FWC) uses empirically known food web links to predict food web links not yet recorded.

A user can choose which food web studies to use for prediction or exclude from 257 datasets we compiled from previously digitized literature.

Taxa can be entered several different ways: simple text lists, XML files, or food web number. In this latter case we seek to reconstruct feeding links based on the rest of the database and can therefore assess the success rate of the different algorithms or model parameters.

Each suspected link is reported, together with references to supporting evidence. Summary statistics of the food web are also reported.

\subsection{Evidence Provider}

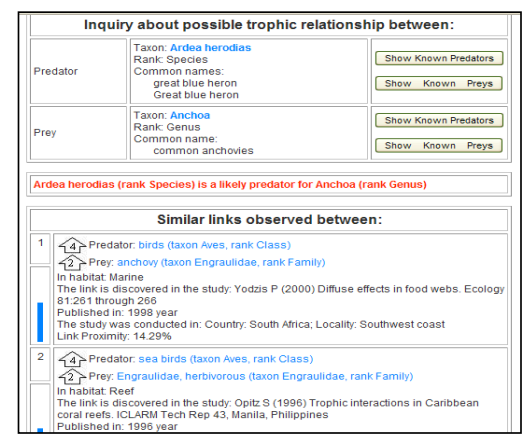

Figure 2. The Evidence Provider allows a user to drill down on a specific link to see the evidence for it.

Figure 2 shows the evidence for a predicted trophic link - namely, the actual link that was observed, the study in which it was published, and the relationship between the species in the observed link and the predicted link.

\subsection{Technical Approach}

The Species List Constructor interacts with web services that provide a variety of species information. Our current Food Web Constructor algorithm uses taxonomic distances to weight evidence supporting or failing to support links between organisms. All data input, output, and taxonomies for Food Web Constructor and Evidence Provider are available in OWL on demand. Calculations are performed on data residing in MySQL databases. Scripts generate OWL documents from Animal Diversity Web (http://www.animaldiversityweb.org) via the ETHAN ontology. The triple shop is currently implemented using the joseki (http://www.joseki.org/).

\section{Swoogle and Triple Shop}

Swoogle (Google for the Semantic Web) is our semantic web search engine [3]. It allows users to search for both ontologies and instance data (collectively referred to as semantic web documents) along a variety of parameters. Once documents are returned, a user can select certain of them for inclusion into her "Triple Shop", a sort of shopping cart for RDF triples. We have built a stand-alone version of the Triple Shop, which allows a user to specify the URLs of arbitrary semantic web documents, and to issue SPARQL queries against the union of those documents.

\subsection{Using the Triple Shop to Integrate Food Web and Natural History Data}

ELVIS illustrates the potential of the semantic web to support rapid querying of distributed scientific databases for a variety of scenarios. For example (Figure 3): Determine known predatorprey relationships among an invader and a specific group of native species in a particular habitat, as reported in previous studies.

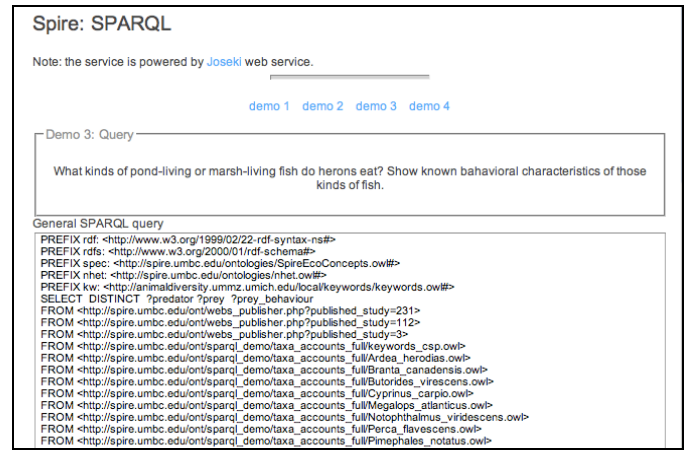

Figure 3. SPARQL query on ETHAN and SpireEcoConcepts OWL documents.

\section{ACKNOWLEDGMENTS}

This research was supported by NSF ITR 0326460 and matching funds received from USGS Nat. Bio. Information Infrastructure.

\section{REFERENCES}

[1] Dunne, J. A. The network structure of food webs. In: Ecological Networks: Linking Structure to Dynamics in Food Webs, eds. Pascual, M. and Dunne, J. A. Oxford University Press, 2005.pp. 27-86.

[2] Jones , M. B.; Berkley, C.; Bojilova, J.; Schildhauer, M. P. 2001. Managing scientific metadata. IEEE Internet Computing. Vol: 5(5). Pages 59-68.

[3] Li Ding et al., "Swoogle: A Search and Metadata Engine for the Semantic Web", Proceedings of the Thirteenth ACM Conference on Information and Knowledge Management, November 2004.

[4] Ludaescher, B. et al. 2004. Scientific workflow management and the Kepler system. Concurrency and Computation: Practice and Experience. 